\title{
Aleitamento materno e níveis de hemoglobina em crianças menores de 2 anos em município do estado de São Paulo, Brasil ${ }^{1}$
}

\author{
Breastfeeding and hemoglobin concentration in \\ children under 2 years of age in a municipality \\ of São Paulo, Brazil
}

\author{
Luciane Simões DUARTE² \\ Elizabeth FUJIMORI² \\ Aurea Tamami MINAGAWA2 \\ Flavia Antonini SCHOEPS 2 \\ Rosali Maria Juliano Marcondes MONTERO ${ }^{3}$
}

\section{RES U M O}

\section{Objetivo}

Avaliar a relação entre aleitamento materno e níveis de hemoglobina em crianças menores de 2 anos residentes na cidade de Itupeva, SP, Brasil.

\section{Métodos}

Estudo transversal de base populacional, realizado em amostra representativa de 254 crianças menores de 2 anos, selecionadas aleatoriamente na área urbana de Itupeva, SP, por procedimento de amostragem por conglomerados em 3 etapas. O aleitamento materno foi classificado utilizando-se as recomendações da Organização Mundial da Saúde e a hemoglobina foi determinada em hemoglobinômetro portátil (HemoCue). Para o diagnóstico, utilizou-se o ponto de corte de 11,0g/dL.

\section{Resultados}

A prevalência de anemia foi de 41,7\%. Anemia foi mais freqüente entre os menores de 6 meses que não se encontravam em aleitamento materno $(p<0,05)$. As médias de hemoglobina das crianças em aleitamento materno também se mostraram significantemente mais elevadas entre os menores de 6 meses $(p<0,05)$. Nessa mesma faixa etária, a média de hemoglobina das crianças em aleitamento materno exclusivo e predominante

\footnotetext{
1 Trabalho financiado pelo Conselho Nacional de Desenvolvimento Científico e Tecnológico (Auxílio pesquisa CNPq - Processo 478872/2004-6).

2 Universidade de São Paulo, Escola de Enfermagem, Departamento de Enfermagem em Saúde Coletiva. Av. Dr. Enéas de Carvalho Aguiar, 419, 05403-000, São Paulo, SP, Brasil. Correspondência para/Correspondence to: E. FUJIMORI. E-mail: <efujimor@usp.br>.

3 Prefeitura Municipal de Itupeva, Secretaria da Saúde. Itupeva, SP, Brasil.
} 
foi significantemente maior do que a observada nas crianças em alimentação artificial $(12,30 \mathrm{~g} / \mathrm{dL}$ versus $10,99 \mathrm{~g} / \mathrm{dL} ; p=0,022$.

\section{Conclusão}

Os resultados encontrados sugeriram que a substituição parcial ou total do leite materno antes dos seis meses de idade associa-se com diminuição dos níveis médios de hemoglobina. A alta prevalência de anemia e o baixo percentual de crianças em aleitamento materno exclusivo chamam atenção para a necessidade do controle dessa deficiência nutricional no município e para a promoção do aleitamento materno exclusivo até os seis meses.

Termos de indexação: aleitamento materno; anemia; fisiologia da nutrição do lactente; hemoglobinas.

\section{A B S T R A C T}

\section{Objective}

The objective of this study is to assess the relationship between breastfeeding and hemoglobin concentration in children under 2 years of age in Itupeva City, SP, Brazil.

\section{Methods}

This cross-sectional population-based study was carried out in a representative sample of 254 children under 2 years of age randomly selected in the urban area of Itupeva, SP. The sampling process was done in three stages. The recommendations made by the World Health Organization were used to classify breastfeeding. Hemoglobin concentration was measured by the cyanmethemoglobin method, using the HemoCue system and anemia was defined when hemoglobin concentration was below 11.0g/dL.

Results

The prevalence of anemia was $41.7 \%$. Anemia was more frequent among children who were weaned before 6 months $(p<0.05)$. The mean hemoglobin levels of breastfed children were significantly higher among children under 6 months $(p<0.05)$. Children who were exclusively or predominantly breastfed in the first six months of life had higher mean hemoglobin levels than the remaining children $(12.3 \mathrm{~g} / \mathrm{dL}$ versus $10.99 \mathrm{~g} / \mathrm{dL}$; $p=0.022$ ).

\section{Conclusions}

The results suggest that partial or total substitution of breast milk before six months of age was associated with lower mean hemoglobin levels. The high frequency of anemia and the low percentage of children who were exclusively breastfed indicate that programs are needed to control nutritional anemia in this city and to promote exclusive breastfeeding in the first six months.

Indexing terms: breast feeding, anemia; infant nutrition physiology; hemoglobins.

\section{N T R O D U ÇÃ O}

A anemia nutricional é definida pela Organização Mundial da Saúde (OMS) como um estado em que a concentração de hemoglobina sangüínea encontra-se anormalmente baixa, em conseqüência da carência de um ou mais nutrientes essenciais ${ }^{1}$. Dessa forma, a medida da concentração de hemoglobina constitui o indicador bioquímico mais utilizado para o diagnóstico da anemia no âmbito populacional².

Embora a falta de vários nutrientes possa determinar a anemia, nas regiões onde sua preva- lência é elevada, a deficiência de ferro é, isoladamente, a causa mais comum ${ }^{3}$. Assim, os termos anemia e anemia ferropriva têm sido usados de forma intercambiável, e, em saúde pública, anemia é sinônimo de anemia ferropriva ${ }^{4}$.

As crianças menores de 2 anos são particularmente vulneráveis à deficiência de ferro, devido ao crescimento acelerado, que impõe maior necessidade orgânica do mineral, aliado ao desmame precoce e baixo consumo de fontes de ferro na alimentação complementar ${ }^{3}$.

No Brasil, não se conhece a distribuição nacional da anemia infantil, porém estudos reali- 
zados em diversas localidades do País revelam prevalências bastante variadas, com indicação de aumento e manutenção em patamares elevados, especialmente entre os menores de 2 anos. Estudos isolados, desenvolvidos em várias regiões do Brasil, mostram as variações e as altas prevalências: $36 \%$ na Paraíba ${ }^{5}$, 50\% no semi-árido baiano $^{6}, 54 \%$ no sul do Brasil 7 , 60\% em Viçosa, $M G^{8,9}, 80 \%$ em Recife, $P E^{10}$. No município de São Paulo, série histórica ${ }^{11}$ comprova que a prevalência da anemia, na faixa etária compreendida entre 6 e 24 meses, praticamente dobrou nas últimas décadas, passando de $35 \%$ na década de $70^{12}$ para $69 \%$ na década de $90^{11}$.

A reconhecida relevância da anemia, em termos de saúde pública, decorre não apenas da magnitude de sua ocorrência, mas, principalmente, dos efeitos deletérios que ocasiona à saúde da criança, tais como repercussões negativas no desenvolvimento psicomotor e cognitivo, diminuição na capacidade de aprendizagem, e comprometimento da imunidade celular, com menor resistência às infecções ${ }^{13,14}$.

Além de a anemia estar associada às precárias condições de vida e às inadequadas condições sanitárias do ambiente em que vivem a criança e sua família, vários fatores contribuem para a diminuição da concentração de hemoglobina sangüínea e o aumento de sua prevalência entre os menores de 2 anos. Destacam-se, entre eles, a idade da criança e o consumo alimentar, tendo papel relevante o desmame precoce e a manutenção de uma alimentação complementar monótona e fundamentalmente láctea, pobre em ferro ${ }^{4,15}$.

A reserva de ferro ao nascer é constituída durante a vida intra-uterina, fase em que o feto necessita manter uma taxa elevada de hemoglobina para compensar a hipóxia da circulação placentária. Após o nascimento ocorre uma hemólise fisiológica, e o ferro liberado da hemoglobina, associado à reserva do mineral feita no último trimestre da gestação, tende a suprir a demanda pelo nutriente nos primeiros meses de vida, em crianças nascidas a termo. Essa condição, normalmente, se dá quando a criança é alimentada exclusivamente com leite materno nos primeiros 6 meses, pois o ferro do leite humano apresenta alta biodisponibilidade, $50 \%$ de seu ferro é absorvido, contra $10 \%$ do ferro do leite de vaca $^{16}$. Tal biodisponibilidade, no entanto, é reduzida com a introdução de outros alimentos ${ }^{17}$.

Estudo realizado nas capitais brasileiras mostra, por sua vez, que a mediana do aleitamento materno em nosso meio é muito baixa, com duração de preocupantes 33 dias ${ }^{18}$. Em outras cidades foram identificados 17 dias em Botucatu ${ }^{19}$, 28 dias em Itupeva ${ }^{20}$ e 39,4 dias no Distrito Federal ${ }^{21}$, embora um estudo tenha encontrado mediana de 120 dias em Lins'22.

Tendo em vista essas considerações, o presente estudo avaliou a relação entre aleitamento materno e níveis de hemoglobina em crianças menores de 2 anos de vida, residentes na cidade de Itupeva, SP, visando a contribuir para a prevenção da anemia nutricional.

\section{M É T O D O S}

Subprojeto de uma investigação mais ampla de base populacional, aprovada pelo Comitê de Ética em Pesquisa da Escola de Enfermagem da Universidade de São Paulo, este estudo transversal, descritivo-analítico foi desenvolvido no município de Itupeva, SP, localizado $70 \mathrm{~km}$ a oeste da cidade de São Paulo. A amostra da investigação mais ampla foi delineada para ser representativa e proporcional ao número de crianças com idade inferior a 2 anos, residentes nos setores censitários que compunham a zona urbana do município.

Utilizando-se o procedimento de amostragem por conglomerados, obteve-se um tamanho amostral de 274 crianças, aceitando-se um erro de até $5 \%$, com previsão de $10 \%$ de perdas e recusas. O sorteio foi realizado em três etapas, obedecendo-se ao critério de probabilidade. Inicialmente foram sorteados os setores censitários, seguido dos conglomerados de domicílios (qua- 
dras), e, finalmente, os domicílios individuais. A amostra final foi constituída por 261 crianças, sendo os dados de hemoglobina e aleitamento, obtidos para 254 crianças.

Os dados foram colhidos nos meses de julho e agosto de 2001, por meio de inquérito domiciliar realizado por enfermeiras e graduandos de enfermagem submetidos a treinamento prévio em técnica de entrevista, coleta de sangue e verificação de medidas de peso e estatura. As informações, obtidas das mães das crianças foram registradas em formulário próprio, pré-testado, contendo questões fechadas.

O perfil de aleitamento materno foi classificado de acordo com as categorias recomenda-das pela Organização Panamericana de Saúde/OMS²3:

- Aleitamento materno (AM): alimentação com leite materno, independentemente do consumo de qualquer complemento, lácteo ou não.

- Aleitamento materno exclusivo (AME): alimentação exclusiva com leite materno, sem qualquer outro líquido ou sólido, porém podendo receber vitaminas, minerais ou medicamentos.

- Aleitamento materno predominante (AMP): alimentação em que o leite materno constitui a principal fonte de alimentação, porém inclui água, chás, suco de frutas, soro de re-hidratação oral, porém nenhum outro leite ou alimento semisólido.

- Aleitamento materno complementado em tempo oportuno: alimentação com leite materno e alimentos sólidos ou semi-sólidos, independentemente da oferta de outro leite ou outros líquidos (lactentes de 6 a 9 meses).

- Aleitamento materno contínuo 1 e 2: lactentes de 12 a 15 meses e de 20 a 23 meses, respectivamente, que continuam recebendo leite materno.

O diagnóstico da anemia foi feito a partir da determinação da concentração de hemoglobina sangüínea pelo método da cianometahemoglobina, utilizando-se o hemoglobinômetro portátil HemoCue, considerado confiável e recomendado pela OMS para avaliação da hemo- globina em estudos epidemiológicos ${ }^{24}$. Realizou-se apenas uma medida de hemoglobina, tendo em vista dificuldades operacionais para coleta de sangue em crianças e considerando não haver diferença entre medidas duplicadas de sangue capilar analisadas em HemoCue ${ }^{25}$.

Crianças com níveis de hemoglobina compatíveis com anemia foram encaminhadas aos serviços de saúde, previamente contatados, para tratamento. Para o diagnóstico, adotou-se o critério da OMS, que define anemia quando a concentração de hemoglobina sangüínea é inferior a $11,0 \mathrm{~g} / \mathrm{dL}^{1}$. Consideraram-se com anemia moderada as crianças com hemoglobina $\geq 9,5 \mathrm{e}<11,0 \mathrm{~g} / \mathrm{dL}$ e grave, com hemoglobina $<9,5 \mathrm{~g} / \mathrm{dL}^{26}$.

Os dados foram armazenados em bancos de dados criados no programa Excel e analisados utilizando-se o programa Epi Info versão 6.04 e o módulo Epi-Nut para avaliação do estado nutricional. O estado nutricional foi caracterizado utilizando-se os índices estatura/idade e peso/estatura, adotando-se como referência o padrão do National Center for Health Statistics ${ }^{27}$, e como ponto de corte para caracterizar desnutrição, -2,0 escore-Z. A análise descritiva foi feita com a construção de tabelas de freqüências uni e bivariadas. As variáveis contínuas foram apresentadas sob a forma de medidas de tendência central (média aritmética) e medida de dispersão (desvio-padrão - DP). Para avaliar associação entre variáveis categóricas utilizou-se o teste do Qui-quadrado, ou teste exato de Fischer, quando necessário; e para comparação estatística entre duas médias utilizou-se o teste " $t$ " de Student. O nível de significância adotado foi de $5 \%(p<0,05)$.

\section{RES ULTA DOS}

A Tabela 1 apresenta as características gerais das crianças estudadas. Constatou-se uma discreta predominância do sexo masculino $(54,7 \%)$ e de menores de 12 meses de idade $(51,2 \%)$. Quanto à duração da gestação e ao peso ao nascer, $8,3 \%$ haviam nascido antes de completar 37 semanas e $6,7 \%$ com menos de $2.500 \mathrm{~g}$. A fre- 
qüência de desnutrição crônica (estatura/idade) e aguda (peso/estatura) foi pequena, 3,5\% e 2,4\%, respectivamente. Mais da metade das mães tinha escolaridade inferior a 8 anos $(51,0 \%)$. A prevalência de anemia para o total da amostra foi de $41,7 \%$, sendo $34,2 \%$ de crianças com anemia moderada (hemoglobina $\geq 9,5$ e $<11 \mathrm{~g} / \mathrm{dL}$ ) e $7,5 \%$ com anemia grave (hemoglobina $<9,5 \mathrm{~g} / \mathrm{dL}$ ).

Do total de crianças estudadas, somente 7 não foram amamentadas, de forma que 97,2\% iniciaram o aleitamento materno. Depreende-se, da Tabela 2, que entre os menores de 4 e 6 meses, $20,5 \%$ e $27,1 \%$, respectivamente, já se encon-

Tabela 1. Características gerais das crianças estudadas. Itupeva, SP, 2001

\begin{tabular}{|c|c|c|}
\hline Variáveis & $\mathrm{n}$ & $\%$ \\
\hline \multicolumn{3}{|l|}{ Sexo } \\
\hline Masculino & 139 & 54,7 \\
\hline Feminino & 115 & 45,3 \\
\hline \multicolumn{3}{|c|}{ Idade (em meses) } \\
\hline$<6$ & 70 & 27,6 \\
\hline $6 \vdash 12$ & 60 & 23,6 \\
\hline $12 \vdash 18$ & 64 & 25,2 \\
\hline$\geq 18$ & 60 & 23,6 \\
\hline \multicolumn{3}{|c|}{ Duração da gestação (em semanas) } \\
\hline$<37$ & 21 & 8,3 \\
\hline$\geq 37$ & 233 & 91,7 \\
\hline \multicolumn{3}{|c|}{ Peso ao nascer ${ }^{1}$ (em gramas) } \\
\hline$<2500$ & 17 & 6,7 \\
\hline$\geq 2500$ & 235 & 93,3 \\
\hline \multicolumn{3}{|c|}{ Altura/ldade (escore-Z) } \\
\hline$<-2$ & 9 & 3,5 \\
\hline$\geq-2$ & 245 & 96,5 \\
\hline \multicolumn{3}{|c|}{ Peso/Altura (escore-Z) } \\
\hline$<-2$ & 6 & 2,4 \\
\hline$\geq-2$ & 248 & 97,6 \\
\hline \multicolumn{3}{|c|}{ Escolaridade materna ${ }^{1}$ (anos de estudo) } \\
\hline $0 \vdash 4$ & 27 & 10,7 \\
\hline $4 \vdash 8$ & 102 & 40,3 \\
\hline $8 \vdash 11$ & 65 & 25,7 \\
\hline$\geq 11$ & 59 & 23,3 \\
\hline \multicolumn{3}{|c|}{ Hemoglobina ( $g / d L)$} \\
\hline$<9,5$ & 19 & 7,5 \\
\hline$\geq 9,5$ e $<11$ & 87 & 34,2 \\
\hline$\geq 11$ & 148 & 58,3 \\
\hline
\end{tabular}

${ }^{1}$ Não foram obtidas informações para a totalidade das crianças.
Tabela 2. Indicadores da situação do aleitamento materno no momento da entrevista. Itupeva, São Paulo, 2001.

\begin{tabular}{lrrrr}
\hline \multirow{2}{*}{ Tipo de AM } & $\begin{array}{c}\text { Faixa etária } \\
\text { (meses) }\end{array}$ & $n$ & \multicolumn{2}{c}{$\begin{array}{c}\text { Crianças } \\
\text { amamentadas }\end{array}$} \\
\cline { 5 - 6 } AM & $0-24$ & 254 & 104 & 40,9 \\
& $<4$ & 44 & 35 & 79,5 \\
AME & $<6$ & 70 & 51 & 72,9 \\
& $<4$ & 44 & 8 & 18,2 \\
AMP & $<6$ & 70 & 9 & 12,9 \\
AM complementado & $6-9$ & 31 & 5 & 16,1 \\
em tempo oportuno & $<6$ & 77 & 14 & 68,2 \\
AM contínuo 1 & $12-15$ & 33 & 5 & 15,2 \\
AM contínuo 2 & $20-24$ & 38 & 12 & 31,6 \\
\hline
\end{tabular}

AM: aleitamento materno; AME: aleitamento materno exclusivo; AMP: aleitamento materno predominante.

travam desmamadas e somente $18,2 \%$ dos menores de 4 meses encontravam-se em AME, enquanto $38,9 \%$ estavam em AMP. Entre os menores de 6 meses, a proporção em AME era de $12,9 \%$ e em AMP, $23,0 \%$.

Observa-se, na Tabela 3, que a anemia era mais freqüente entre as crianças menores de 6 meses que não se encontravam em aleitamento materno no momento da entrevista $(p=0,031)$. Constata-se também que os valores médios de hemoglobina das crianças com menos de 6 meses, em aleitamento materno, eram sistematicamente mais elevados $(p=0,041)$.

Verifica-se, pela Tabela 4, que as crianças menores de 6 meses em aleitamento materno apresentavam média de hemoglobina discretamente superior em relação àquelas desmamadas, porém diferença estatisticamente significante foi observada somente entre as crianças em AMP $(p=0,004)$.

Considerando que apenas 9 crianças encontravam-se em AME, procedeu-se à análise dos dados agrupando as crianças menores de 6 meses, em AME e em AMP, ou seja, crianças em que o leite materno constitui a principal fonte de alimentação, porém também inclui água, chás ou suco de frutas, mas nenhum outro leite ou 
Tabela 3. Distribuição da presença ou não de anemia e médias $(M)$ de hemoglobina $(\mathrm{g} / \mathrm{dL})$, segundo situação do aleitamento materno no momento da entrevista e faixa etária. Itupeva, SP, 2001.

\begin{tabular}{|c|c|c|c|c|c|c|c|c|c|}
\hline \multirow{5}{*}{$\begin{array}{c}\text { Faixa etária- } \\
\text { (meses) }\end{array}$} & \multicolumn{8}{|c|}{ Aleitamento materno } & \multirow{5}{*}{$p$} \\
\hline & \multicolumn{4}{|c|}{ Sim } & \multicolumn{4}{|c|}{ Não } & \\
\hline & \multicolumn{2}{|c|}{ Anêmicos } & \multicolumn{2}{|c|}{ Não anêmicos } & \multicolumn{2}{|c|}{ Anêmicos } & \multicolumn{2}{|c|}{ Não anêmicos } & \\
\hline & $\mathrm{n}$ & $\%$ & $\mathrm{n}$ & $\%$ & $n$ & $\%$ & $\mathrm{n}$ & $\%$ & \\
\hline & \multicolumn{4}{|c|}{$\mathrm{Hb} \mathrm{M} \quad \mathrm{DP}^{1}$} & \multicolumn{4}{|c|}{$\mathrm{Hb} M \quad \mathrm{DP}^{1}$} & \\
\hline \multirow[t]{2}{*}{$<6$} & 13 & 25,5 & 38 & 74,5 & 10 & 52,6 & 9 & 47,4 & $0,031^{2}$ \\
\hline & & 11,86 & 1,64 & & & 11,09 & 1,12 & & $0,041^{3}$ \\
\hline \multirow[t]{2}{*}{$6 \vdash 12$} & 17 & 63,0 & 10 & 37,0 & 17 & 51,5 & 16 & 48,5 & $0,373^{2}$ \\
\hline & & 10,80 & 1,01 & & & 11,00 & 1,29 & & $0,494^{3}$ \\
\hline \multirow[t]{2}{*}{$\geq 12$} & 12 & 44,5 & 15 & 55,5 & 37 & 38,2 & 60 & 61,8 & $0,554^{2}$ \\
\hline & & 11,03 & 1,15 & & & 11,35 & 1,37 & & $0,332^{3}$ \\
\hline
\end{tabular}

'Desvio-padrão; ${ }^{2}$ Teste do Qui-quadrado; ${ }^{3}$ Teste " $t$ " de Student.

Tabela 4. Distribuição das médias (M) de hemoglobina ( $\mathrm{g} / \mathrm{dL}$ ), segundo situação do aleitamento materno no momento da entrevista e regimes de aleitamento. Itupeva, SP, 2001

\begin{tabular}{|c|c|c|c|c|c|c|c|c|}
\hline \multirow{2}{*}{ Regimes de aleitamento } & \multirow{2}{*}{$\begin{array}{l}\text { Faixa etária } \\
\text { (meses) }\end{array}$} & \multicolumn{3}{|c|}{ Amamentadas } & \multicolumn{3}{|c|}{ Desmamadas } & \multirow{2}{*}{$p^{2}$} \\
\hline & & $\mathrm{n}$ & $\mathrm{Hb} \mathrm{M}$ & $\mathrm{DP}^{1}$ & $\mathrm{n}$ & $\mathrm{Hb} \mathrm{M}$ & $\mathrm{DP}^{1}$ & \\
\hline AME & $<6$ & 9 & 11,26 & 1,45 & 19 & 11,09 & 1,12 & 0,902 \\
\hline AMP & $<6$ & 14 & 12,98 & 2,01 & 19 & 11,09 & 1,12 & 0,005 \\
\hline $\begin{array}{l}\text { AM Complementado } \\
\text { em tempo oportuno }\end{array}$ & $6-9$ & 5 & 10,40 & 0,79 & 16 & 10,88 & 1,06 & 0,321 \\
\hline AM Contínuo 1 & $12-15$ & 5 & 10,60 & 1,38 & 28 & 11,00 & 1,31 & 0,513 \\
\hline AM Contínuo 2 & $20-24$ & 12 & 11,05 & 1,32 & 26 & 11,70 & 1,58 & 0,292 \\
\hline
\end{tabular}

${ }^{1}$ DP: desvio-padrão; ${ }^{2}$ Teste " $t$ " de Student.

Tabela 5. Distribuição das médias ( $M$ ) de hemoglobina ( $\mathrm{g} / \mathrm{dL}$ ) de crianças menores de 6 meses, segundo regime de aleitamento. Itupeva, SP, 2001.

\begin{tabular}{llcccr}
\hline \multirow{2}{*}{ Regimes de aleitamento } & $\mathrm{n}$ & \multicolumn{2}{c}{ Hemoglobina $(\mathrm{g} / \mathrm{dL})$} & \multirow{2}{*}{$p^{2}$} \\
\cline { 3 - 4 } & & $\mathrm{M}$ & $\mathrm{DP}$ & \\
\hline AME + AMP & 23 & 12,30 & 1,98 & \\
Aleitamento misto & 29 & 11,37 & 1,03 & 0,110 \\
Aleitamento artificial & 21 & 10,99 & 1,14 & 0,022 \\
\hline
\end{tabular}

${ }^{1} \mathrm{DP}$ : desvio-padrão; ${ }^{4}$ Teste " $t$ " de Student entre AME+AMP e Aleitamento misto; e entre AME+AMP e Aleitamento artificial; ${ }^{3}$ Aleitamento materno exclusivo + Aleitamento materno predominante.

alimento semi-sólido ou sólido. A Tabela 5 relaciona os valores médios de hemoglobina das crianças menores de 6 meses com esse regime alimentar (AME + AMP), versus crianças em aleitamento misto (crianças com leite materno e outros tipos de leite) e em alimentação artificial (crianças recebendo somente outros tipos de leite, não o materno). Verificou-se que as médias de hemoglobina das crianças em AME/AMP eram significantemente maiores em relação às das crianças em aleitamento artificial $(p<0,05)$.

\section{I S C U S S Ã O}

Os resultados obtidos no presente estudo revelam que a prevalência de anemia é bastante elevada entre os menores de 2 anos do município de Itupeva, SP, superando em mais de 10 vezes a proporção de crianças afetadas pela desnutrição crônica, ou seja, com déficit estatural (estatura/ idade $<-2$ escore-Z). Segundo a $\mathrm{OMS}^{3}$, prevalências de anemia superiores a $40 \%$ se caracterizam como um grave problema de saúde pública, 
situação que tem sido reiteradamente constatada em nosso meio, e também confirmada em Itupeva ${ }^{7,28}$.

Acresce-se a isso, a acurácia dos exames realizados em hemoglobinômetros portáteis. Estudo de validação da medida da hemoglobina em HemoCue mostrou que a especificidade para o diagnóstico da anemia é adequada (>90,0\%), porém a sensibilidade é relativamente baixa $(<80,0 \%)$. Ademais, esse estudo constatou um erro sistemático de $+0,5 \mathrm{~g} / \mathrm{dL}$ na medida da concentração de hemoglobina em sangue capilar, comparado ao sangue venoso ${ }^{25}$. Ajustando-se os valores de hemoglobina para esse erro, a prevalência de anemia na amostra estudada seria ainda mais elevada, passando para 59,4\%.

Entre as crianças estudadas, quase $100,0 \%$ iniciaram o AM, porém, aos 6 meses, mais de um quarto já se encontravam desmamadas, apesar da recomendação de que o AME seja mantido até essa idade e o aleitamento materno, acrescido de alimentos complementares, mantido até os dois anos de idade ou mais².

Para os menores de 4 e 6 meses do estudo, o percentual de crianças em AM - 79,5\% e 72,9\%, respectivamente; em AME - 18,2\% e 12,9\%, respectivamente, foi inferior ao encontrado por Vieira et al. ${ }^{29} \mathrm{em}$ Feira de Santana, BA. Nessa localidade, 87,9\% das crianças com menos de 4 meses ainda mamavam e, dessas, 45,6\% encontravam-se em AME, enquanto para os menores de 6 meses, as proporções foram 82,2\% em AM e $36,9 \%$ em AME.

Analisando-se somente a proporção de 18,2\% de crianças em AME aos 4 meses, os resultados de outros estudos são bastante divergentes, 16,0\% em Botucatu ${ }^{19}$, 5,8\% em Porto Alegre $^{30}$ e 8,0\% em Montes Claros ${ }^{31}$. Constata-se, assim, que a situação do AME em Itupeva é tão preocupante quanto em várias outras localidades brasileiras.

Sabe-se que a curta duração do AME tem sido apontada como um dos principais fatores que contribuem para a diminuição dos níveis de hemoglobina entre os lactentes, uma vez que, mesmo contendo quantidades reduzidas de ferro, a biodisponibilidade do mineral presente no leite humano é bastante elevada. Contudo, tal biodisponibilidade pode reduzir-se em até $80 \%$, quando novos alimentos são introduzidos na alimentação da criança $^{15}$

Os dados do presente estudo confirmaram associação estatisticamente significante entre aleitamento materno e anemia em crianças menores de 6 meses. Da mesma forma que Torres et al. ${ }^{28}$, constatou-se que mais da metade das crianças não amamentadas durante os primeiros 6 meses desenvolvia anemia. Esses autores verificaram que entre crianças não amamentadas, $60,6 \%$ das menores de 2 meses eram anêmicas, proporção que praticamente se manteve na faixa etária de 3-6 meses $(57,6 \%)$ e entre os maiores de 6 meses (55,5\%).

Szarfarc et al. ${ }^{32}$, por sua vez, também observaram menor prevalência de anemia entre crianças em AM (45,8\%), seguida por aquelas com alimentação mista $(59,6 \%)$ e artificial $(62,5 \%)$.

A análise dos níveis médios de hemoglobina, conforme a presença ou não de aleitamento materno, mostrou associação significante somente para os menores de 6 meses, de forma que as médias de hemoglobina das crianças em aleitamento eram significantemente maiores que das crianças desmamadas. Esse dado ratifica a importância do AM como fator de proteção contra anemia entre os menores de 6 meses.

Ao avaliar as médias de hemoglobina segundo os regimes de $\mathrm{AM}$, verificou-se diferença estatisticamente significante somente para as crianças em AMP, ou seja, a média de hemoglobina das crianças com esse regime alimentar era significantemente maior em relação às médias daquelas que não se encontravam com esse tipo de aleitamento. Possivelmente, essa associação não foi verificada para as crianças em AME, devido ao reduzido número de casos. Entre os maiores de 6 meses, tal associação não seria esperada, uma vez, que após essa idade, o aleitamento materno deixa de ser o principal componente ali- 
mentar, passando à alimentação complementar o papel preponderante na prevenção da anemia.

Em estudo da relação entre os níveis médios de hemoglobina e o consumo de leite materno, Assis et al.$^{33}$ encontraram concentrações mais elevadas de hemoglobina entre as crianças em AME, seguidas por aquelas que se encontravam em AMP. Os resultados mais desvantajosos foram observados entre as crianças em regime de aleitamento materno complementado, seguido, daquelas em aleitamento artificial complementado, indicando que a alimentação complementar oferecida à criança não tem sido adequada para garantir o aporte de ferro.

Também neste estudo, crianças menores de 6 meses em aleitamento materno exclusivo ou predominante apresentaram médias de hemoglobina estatisticamente superiores às das crianças em aleitamento artificial.

\section{O N CLUS Ã O}

Como subprojeto de uma investigação mais ampla, que teve como finalidade caracterizar os determinantes das condições de saúde e nutrição da população menor de dois anos da cidade de Itupeva, este estudo buscou avaliar a relação entre aleitamento materno e níveis de hemoglobina em crianças menores de 2 anos, considerando que a $\mathrm{OMS}^{24}$ recomenda seu prolongamento até essa faixa etária, acrescido de alimentos complementares após o sexto mês de vida.

Os resultados encontrados sugeriram uma associação entre presença de aleitamento materno e maiores níveis de hemoglobina somente entre os menores de 6 meses, como esperado, uma vez que após essa idade a influência do aleitamento materno sobre a ocorrência da anemia é mínima. Entretanto, considerando a magnitude da problemática da anemia e a abrangência de seus determinantes, não analisadas no presente estudo, não é possível atribuir o desenvolvimento da anemia, e/ou os menores níveis de hemo- globina, ao abandono precoce do aleitamento materno.

O baixo percentual de crianças em AME verificado no município de Itupeva, evidencia a necessidade de concentrar esforços no sentido de promover a manutenção do aleitamento materno exclusivo até os 6 meses e, a partir dessa idade, sua manutenção, acrescida de alimentos complementares quali-quantitativamente adequados, como recomendado pela $\mathrm{OMS}^{24}$, estratégia que, certamente, irá contribuir para elevar os níveis médios de hemoglobina dos lactentes e prevenir o desenvolvimento da anemia.

\section{A GRADECIMENTOS}

À Prefeitura Municipal de Itupeva, às mães e crianças que aceitaram participar do estudo, à Coordenação de Aperfeiçoamento de Pessoal de Nível Superior, pelas bolsas de mestrado concedidas e ao Conselho Nacional de Desenvolvimento Ciênctífico e Tecnológico, pelo auxílio financeiro e bolsas de iniciação científica concedidos.

\section{REFERÊ NCIAS}

1. Organización Mundial de la Salud. Anemias nutricionales. Ginebra; 1968. Série de Informes Técnicos, 405.

2. World Health Organization. The optimal duration of exclusive breastfeeding. Note for press $n^{\circ} 7$, 2001. Available from: http://www.who.int/inf-pr2001/en/note2001-07.html

3. Administrative Committee on Coordination. Sub Committee on Nutrition. Third Report on the World Nutrition Situation. Geneva; 1997.

4. Szarfarc SC. Anemia ferropriva e saúde pública. Pediatr Mod. 1993; 29(1):39-43.

5. Oliveira RS, Diniz AS, Benigna MJC, Miranda-Silva SM, Lola MM, Gonçalves MC, et al. Magnitude, distribuição espacial e tendência da anemia em pré-escolares da Paraíba. Rev Saúde Pública. 2002; 36(1):26-32.

6. Assis AMO, Santos, LMP, Martins MC, Araújo MPN, Amorim DQ, Morris SS, et al. Distribuição da anemia em pré-escolares do semi-árido da Bahia. Cad Saúde Pública. 1997; 12(2):237-43. 
7. Neumann NA, Tanaka OY, Szarfarc SC, Guimarães PRV, Victoria CG. Prevalência e fatores de risco para anemia no sul do Brasil. Rev Saúde Pública. 2000; 34(1):57-63.

8. Silva DG, Franceschini SCC, Priore SE, Ribeiro SMR, Szarfarc SC, Souza SB, et al. Anemia ferropriva em crianças de 6 a 12 meses atendidas na rede pública de saúde do município de Viçosa, Minas Gerais. Rev Nutr. 2002; 15(3):301-8.

9. Miranda AS, Franceschini SCC, Priore SE, Euclydes MP, Araújo RMA, Ribeiro SMR, et al. Anemia ferropriva e estado nutricional de crianças com idade de 12 a 60 meses do município de Viçosa, MG. Rev Nutr. 2003; 16(2):163-9.

10. Osório MM, Lira PIC, Batista-Filho M, Ashworth A. Prevalence of anemia in children 6-59 months old in the state of Pernambuco, Brazil. Rev Panam Salud Pública. 2001; 10(2):101-7.

11. Monteiro CA, Szarfarc SC, Mondini L. Tendência secular da anemia na infância na cidade de São Paulo (1984-1996). Rev Saúde Pública. 2000; 34(6 Supl):62-72.

12. Sigulem DM, Tudisco ES, Goldenberg P, Athaide MMM, Vaisman E. Anemia ferropriva em crianças do município de São Paulo. Rev Saúde Pública. 1978; 12(2):168-79

13. Walter T, Olivares M, Pizarro F, Muñoz C. Iron, anemia and infection. Nutr Rev. 1997; 55(4):111-24.

14. Pollitt E. Early iron deficiency anemia and later mental retardation. Am J Clin Nutr. 1999; 69(1): 4-5.

15. Osório MM. Fatores determinantes da anemia em crianças. J Pediatr. 2002; 78(4):269-78.

16. Giugliani ERJ, Victora CG. Normas alimentares para crianças brasileiras menores de dois anos: bases científicas. Brasília: Organização Panamericana da Saúde; 1997.

17. Dewey KG, Cohen REJ, Rivera LL, Brown KH. Effects of age of introduction of complementary foods on iron status of breast-fed infants in Honduras. Am J Clin Nutr. 1998; 67:878-84.

18. Brasil. Ministério da Saúde. Secretaria de Políticas de Saúde. Área de Saúde da Criança. Prevalência do AM nas capitais brasileiras e no Distrito Federal. Brasília; 2001.

19. Carvalhaes MABL, Parada CMGL, Manoel CM, Venâncio SI. Diagnóstico da situação do aleitamento materno em área urbana do sudeste do Brasil: utilização de metodologia simplificada. Rev Saúde Pública. 1998; 32(5):430-6.

20. Minagawa AT, Oliveira IMV, Fujimori E, Laurenti E, Montero RMJM. Perfil do aleitamento materno em menores de 2 anos na cidade de Itupeva, SP, Brasil. Arch Latinoam Nutr. 2005; 55(2):132-9.
21. Sena MCF, Silva EF, Pereira MG. Prevalência do aleitamento materno no Distrito Federal, Brasil. Cad Saúde Pública. 2002; 18(3):613-21.

22. Silva AP Souza N. Prevalência do aleitamento materno. Rev Nutr. 2005; 18(3):301-10.

23. Organización Panamericana de la Salud. Indicadores para evaluar las practicas de lactancia materna. Genebra: OMS; 1991

24. World Health Organization. Iron deficiency anemia: assessment, prevention and control: a guide for programme managers. Geneva; 2001.

25. Neufeld L, Garcia-Guerra A, Sánchez-Francia D, Newton- Sánchez O, Ramirez-Villalobos MD, et al. Hemoglobin measured by HemoCue and reference method in venous and capillary blood: a validation study. Salud Publica Mex. 2002; 44(3):219-27.

26. Szarfarc SC, Berg G, Santos ALS, Souza SB, Monteiro CA. Prevenção de anemia no primeiro ano de vida em centros de saúde do município de Santo André, São Paulo. J Pediatr. 1996; 72(5):329-34.

27. Hamill PVV, Drizd TA, Johnson CL, Reed RB, Roche AF. NCHS growth curves for children. Birth - 18 years, United States. Washington (DC): National Center for Health Statistics; 1977. DHEW Publication $\mathrm{n}^{\circ}$ (PHS) 78-1650.

28. Torres MAA, Sato K, Queiroz SS. Anemia em crianças menores de dois anos atendidas nas unidades básicas de saúde no Estado de São Paulo Brasil. Rev Saúde Pública. 1994; 28(4):290-4.

29. Vieira GO, Glisser M, Araújo SPT, Sales NA Indicadores do aleitamento materno na cidade de Feira de Santana, Bahia. J Pediatr. 1998; 74(1):11-6.

30. Kummer SC, Giugliani ERJ, Susin O, Folleto JL, Lermen NR, Santos L, et al. Evolução do padrão de aleitamento materno. Rev Saúde Pública. 2000; 34(2):143-8.

31. Caldeira AP, Goulart EMA. A situação do aleitamento materno em Montes Claros, Minas Gerais: estudo de uma amostra representativa. J Pediatr. 2000; 76(1):65-72.

32. Szarfarc SC, Souza SB, Furumoto RAV, Brunken GS, Assis AMO, Galdenzi EM, et al. Concentração de hemoglobina em crianças do nascimento até um ano de vida. Cad Saúde Pública. 2004; 20(1): 266-74.

33. Assis AMO, Gaudenzi EM, Gomes G, Ribeiro RC, Szarfarc SC, Souza SB. Níveis de hemoglobina, aleitamento materno e regime alimentar no primeiro ano de vida. Rev Saúde Pública. 2004; 38(4):543-51.

Recebido em: 17/1/2006

Versão final reapresentada em: 30/10/2006

Aprovado em: 21/11/2006 
